Original article

\title{
Integral transforms and extended Voigt functions
}

\author{
(D) M.A. Pathan
}

\author{
Centre for Mathematical and Statistical Sciences, Peechi P.O., Kerala-680653, India
}

\begin{abstract}
In this paper we introduce a generalization of the Voigt functions and discuss their properties and applications. Some interesting explicit series representations, integrals and identities and their link to Jacobi,Laguerre and Hermite polynomials are obtained. The resulting formulas allow a considerable unification of various special results which appear in the literature. (C) 2019. Acad. Colomb. Cienc. Ex. Fis. Nat.
\end{abstract}

Key words: Voigt function; Bessel function; Parabolic function; Hypergeometric function and Laguerre polynomials.

Transformadas integrales y funciones extendidas de Voigt

\section{Resumen}

En este artículo se introduce una generalización de las funciones de Voigt y se discuten sus propiedades y aplicaciones. Se obtienen representaciones explícitas de series, integrales e identidades y sus conexiones con los polinomios de Jacobi, Laguerre y Hermite. Las fórmulas resultantes permiten la unificación de algunos resultados especiales que aparecen en la literatura. (C) 2019. Acad. Colomb. Cienc. Ex. Fis. Nat.

Palabras clave: Función de Voigt; Función de Bessel; Función parabólica; Polinomio de Laguerre.

\section{Introduction}

The Voigt functions $K(x ; y)$ and $L(x ; y)$ are effective tools for solving a wide variety of problems in probability, statistical communication theory, astrophysical spectroscopy, emission, absorption and transfer of radiation in heated atmosphere, plasma dispersion, neutron reactions and indeed in the several diverse field of physics and engineering associated with multi-dimensional analysis of spectral harmonics. The Voigt functions are natural consequences of the well-known Hankel transforms, Fourier transforms and Mellin transforms, resulting in connections with the special functions. Many mathematicians and physicists have contributed to a better understanding of these functions.For a number of generalizations of Voigt functions,we refer Yang (1994). Pathan, et al. (2003), (2006), Klusch (1991) and Srivastava, et al. (1998). Following the work of Srivastava, et al. (1987), Klusch (1991) has given a generalization of the Voigt functions in the form

$$
\begin{aligned}
& V_{\mu, \nu}(x, y, z)=\sqrt{\frac{x}{2}} \int_{0}^{\infty} t^{\mu} e^{-y t-z t^{2}} J_{\nu}(x t) d t \\
&= \frac{z^{-\alpha} x^{\nu+\frac{1}{2}}}{2^{\nu+\frac{1}{2}} \Gamma(\nu+1)}\left\{\Gamma(\alpha) \psi_{2}\left[\alpha ; \nu+1, \frac{1}{2} ;-\frac{x^{2}}{4 z},-\frac{y^{2}}{4 z}\right]\right. \\
&-\left.\frac{y}{\sqrt{z}} \Gamma\left(\alpha+\frac{1}{2}\right) \psi_{2}\left[\alpha+\frac{1}{2} ; \nu+1, \frac{3}{2} ;-\frac{x^{2}}{4 z},-\frac{y^{2}}{4 z}\right]\right\} \\
&\left(\alpha=(\mu+\nu+1) / 2, x, y, z \in \mathbb{R}^{+}, R(\mu+\nu)>-1\right)
\end{aligned}
$$

where $\psi_{2}$ denotes one of Humbert's confluent hypergeometric function of two variables, defined by Srivastava, et al. (1984), p.59

$\psi_{2}\left[\alpha ; \gamma, \gamma^{\prime} ; x, y\right]=\sum_{m, n=0}^{\infty} \frac{(\alpha)_{m+n}}{(\gamma)_{m}\left(\gamma^{\prime}\right)_{n}} \frac{x^{m} y^{n}}{m ! n !}, \max \{|x|,|y|\}<\infty$,

$(\lambda)_{n}$ being the Pochhammer symbol defined (for $\lambda \in \mathbb{C}$ ) by

$$
\begin{gathered}
(\lambda)_{n}:=\lambda(\lambda+1) \ldots(\lambda+n-1)(n \in \mathbb{N}) \\
=\frac{\Gamma(\lambda+n)}{\Gamma(\lambda)} \quad\left(\lambda \in \mathbb{C} \backslash \mathbb{Z}_{0}^{-}\right) .
\end{gathered}
$$

The classical Bessel function $J_{v}(x)$ is defined by (see, Andrews, et al. (1999)).

$$
J_{\nu}(z)=\sum_{m=0}^{\infty} \frac{(-1)^{m}\left(\frac{z}{2}\right)^{\nu+2 m}}{m ! \Gamma(\nu+m+1)},(z \in \mathbb{C} \backslash(-\infty, 0))
$$

so that

$$
\begin{array}{r}
K(x, y)=V_{1 / 2,-1 / 2}\left(x, y, \frac{1}{4}\right) \\
\text { and } L(x, y)=V_{1 / 2,1 / 2}\left(x, y, \frac{1}{4}\right)
\end{array}
$$

Corresponding autor:

M.A.Pathan; mapathan@gmail.com

Received: Octubre 11, 2018

Accepted: April 4, 2019

Editor: Jorge Cossio Betancur 
Observe that $J_{v}(z)$ is the defining oscillatory kernel of Hankel's integral transform

$$
\left(H_{\nu} f\right)(x)=\int_{0}^{\infty} f(t) J_{\nu}(x t) d t
$$

${ }_{p} F_{q}$ is the generalized hypergeometric series defined by (see, Andrews, et al. (1999)).

$$
\begin{aligned}
& { }_{p} F_{q}\left[\begin{array}{c}
\left(\alpha_{1}\right),\left(\alpha_{2}\right), \ldots,\left(\alpha_{p}\right) ; z \\
\left(\beta_{1}\right),\left(\beta_{2}\right), \ldots,\left(\beta_{q}\right) ;
\end{array}\right] \\
& =\sum_{n=0}^{\infty} \frac{\left(\alpha_{1}\right)_{n} \ldots\left(\alpha_{p}\right)_{n}}{\left(\beta_{1}\right)_{n} \ldots\left(\beta_{q}\right)_{n}} \frac{z^{n}}{n !} \\
& ={ }_{p} F_{q}\left(\alpha_{1}, \ldots, \alpha_{p} ; \beta_{1}, \ldots, \beta_{q} ; z\right)
\end{aligned}
$$

The following hypergeometric representation for the Jacobi polynomials $P_{n}^{\alpha, \beta}(x)$ is a special case of the above generalized hypergeometric series

$$
\begin{aligned}
& P_{n}^{\alpha, \beta}(x)=(-1)^{n} P_{n}^{\alpha, \beta}(-x)= \\
& \frac{(\alpha+1)_{n}}{n !}{ }_{2} F_{1}\left(-n, \alpha+\beta+n+1 ; \alpha+1 ; \frac{1-x}{2}\right)
\end{aligned}
$$

Another special case [Prudnikov, et al. (1986), p.579 (18)] expressible in terms of hypergeometric function is

$$
\begin{gathered}
{ }_{1} F_{1}(-n, \alpha+1 ; z)=\frac{n !}{(\alpha+1)_{n}} L_{n}^{(\alpha)}(z)= \\
\sum_{k=0}^{n} \frac{(\alpha+1)_{n}}{(n-k) ! k ! \alpha+1)_{k}}\left(-z^{k}\right)
\end{gathered}
$$

where $L_{n}^{(\alpha)}(z)$ is Laguerre polynomial [Andrews, et al. (1999)].

The generalized Hermite polynomials (known as GouldHopper polynomials) $H_{n}^{r}(x ; y)$ [Gould, et al. (1962)] defined by

$$
e^{x t+y t^{r}}=\sum_{n=0}^{\infty} H_{n}^{r}(x, y) \frac{t^{n}}{n !}
$$

are 2-variable Kampe de Feriet generalization of the Hermite polynomials Dattoli, et al. (2003) and Gould, et al. (1962)

$$
H_{n}(x, y)=n ! \sum_{r=0}^{\left[\frac{n}{2}\right]} \frac{y^{r} x^{n-2 r}}{r !(n-2 r) !}
$$

These polynomials usually defined by the generating function

$$
e^{x t+y t^{2}}=\sum_{n=0}^{\infty} H_{n}(x, y) \frac{t^{n}}{n !}
$$

reduce to the ordinary Hermite polynomials $H_{n}(x)$ (when $y=$ -1 and $x$ is replaced by $2 x$ ).
We recall that the Hermite numbers $H_{n}$ are the values of the Hermite polynomials $H_{n}(x)$ at zero argument that is $H_{n}(0)$ $=0$. A closed formula for $H_{n}$ is given by

$$
H_{n}= \begin{cases}0, & \text { if } \mathrm{n} \text { is odd } \\ \frac{(-1)^{n / 2} n !}{\left(\frac{n}{2}\right) !}, & \text { if } \mathrm{n} \text { is even }\end{cases}
$$

Altin, et al. (2006) presented a multivariable extension of the so called Lagrange-Hermite polynomials generated by [see Altin, et al. (2006), p.239, Eq.(1.2)] and Chan, et al. (2001):

$$
\begin{aligned}
& \prod_{j=1}^{r}\left(1-x_{j} t^{j}\right)^{-\alpha_{j}}=\sum_{n=0}^{\infty} h_{n}^{\left(\alpha_{1}, \cdots, \alpha_{r}\right)}\left(x_{1}, \cdots x_{r}\right) t^{n} \\
& \left(\alpha_{j} \in \mathbb{C}(j=1, \cdots, r)\right) ;|t|<\min \left\{\left|x_{1}\right|^{-1},\left|x_{2}\right|^{-\frac{1}{2}}, \cdots,\left|x_{r}\right|^{-\frac{1}{r}}\right\} \\
& \text { where } \\
& h_{n}^{\left(\alpha_{1}, \cdots, \alpha_{r}\right)}\left(x_{1}, \cdots, x_{r}\right)=\sum_{k_{1}+2 k_{2}+\cdots+r k_{r}=n}\left(\alpha_{1}\right)_{k_{1}} \cdots\left(\alpha_{r}\right)_{k_{r}} \frac{x_{1}^{k_{1}}}{k_{1} !} \cdots \frac{x_{r}^{k_{r}}}{k_{r} !}
\end{aligned}
$$

The special case when $r=2$ in (1.13) is essentially a case which corresponds to the familiar (two-variable) LagrangeHermite polynomials $h_{n}^{\left(\alpha_{1}, \alpha_{2}\right)}\left(x_{1} ; x_{2}\right)$ considered by Dattoli, et al. (2003)

$\left(1-x_{1} t\right)^{-\alpha_{1}}\left(1-x_{2} t^{2}\right)^{-\alpha_{2}}=\sum_{n=0}^{\infty} h_{n}^{\left(\alpha_{1}, \alpha_{2}\right)}\left(x_{1}, x_{2}\right) t^{n}$

The present work is inspired by the frequent requirements of various properties of Voigt functions in the analysis of certain applied problems. In the present paper it will be shown that generalized Voigt function is expressible in terms of a combination of Kampe de Feriet's functions. We also give further generalizations (involving multivariables) of Voigt functions in terms of series and integrals which are specially useful when the parameters take on special values. The results of multivariable Hermite polynomials are used with a view to obtaining explicit representations of generalized Voigt functions. Our aim is to further introduce two more generalizations of (1.1) and another interesting explicit representation of (1.1) in terms of Kampe de Feriet series $F_{l: m ; n}^{p: q ; r}[$ see (Srivastava, et al. (1984), p.63)]. Finally we discuss some useful consequences of Lagarange-Hermite polynomials and analyze the relations among different generalized Voigt functions.

\section{Generalized Voigt function $\Phi_{\mu, v, r}^{\alpha, \beta}$}

In an attempt to generalize (1.1), we first investigate here the generalized Voigt function $\Phi_{\mu, v, r}^{\alpha, \beta}$

Denition The generalized Voigt function $\Phi_{\mu, v, r}^{\alpha, \beta}$ is defined by the Hankel transform

$$
\begin{gathered}
\Phi_{\mu, v, r}^{\alpha, \beta}=\Phi_{\mu, v, r}^{\alpha, \beta}(x, y, z \mid X) \\
=\sqrt{\frac{x}{2} \int_{0}^{\infty} t^{\mu} e^{-y t-z t^{r}}{ }_{1} F_{1}(\alpha+\beta+1, \alpha+1 ;-X y t)_{1}} \\
F_{1}\left(\alpha+\beta+1, \alpha+1 ;-X z t^{r}\right) J_{\nu}(x t) d t
\end{gathered}
$$

where $x ; y ; z ; X \in \mathbb{R}^{+}, r>1, \operatorname{Re}(\alpha)>-1$ and $\operatorname{Re}(\mu+v>-1$. 
A fairly wide variety of Voigt functions can be represented in terms of the special cases of (2.1). We list below some cases.

The generalized Voigt function

$$
\Phi_{\mu, v, 2}^{\alpha, \beta}=\mathbf{\Omega}_{\mu, v}^{\alpha, \beta}=\Omega_{\mu, v}^{\alpha, \beta}(x, y, z \mid X)
$$

is defined by the integral representation

$$
\begin{aligned}
& \Omega_{\mu, v}^{\alpha, \beta}(x, y, z \mid X)= \\
& \sqrt{\frac{x}{2} \int_{0}^{\infty} t^{\mu} e^{-y t-z t^{2}}{ }_{1} F_{1}(\alpha+\beta+1, \alpha+1 ;-X y t)_{1}} \\
& \quad F_{1}\left(\alpha+\beta+1, \alpha+1 ;-X z t^{2}\right) J_{v}(x t) d t
\end{aligned}
$$

where $x, y, z, X \in \mathbb{R}^{+}, \operatorname{Re}(\alpha)>-1$ and $\operatorname{Re}(\mu+v>-1$.

An obvious special case of (2.1) occurs when we take $r=2$ and $X=1$. We thus have

$$
\begin{aligned}
& \omega_{\mu, v}^{\alpha, \beta}(x, y, z)=\left.\Omega_{\mu, v}^{\alpha, \beta}(x, y, z \mid X)\right|_{X=1} \\
& =\sqrt{\frac{x}{2} \int_{0}^{\infty} t^{\mu} e^{-y t-z t^{2}}{ }_{1} F_{1}(\alpha+\beta+1, \alpha+1 ;-y t){ }_{1}} \\
& \quad F_{1}\left(\alpha+\beta+1, \alpha+1 ;-z t^{2}\right) J_{v}(x t) d t
\end{aligned}
$$

Clearly, the case $X=0$ in (2.1) reduces to a generalization of (1.1) in the form

$$
\begin{aligned}
V_{\mu, v, r}(x, y, z) & =\Phi_{\mu, v, r}^{\alpha, \beta}(x, y, z \mid 0) \\
& =\sqrt{\frac{x}{2}} \int_{0}^{\infty} t^{\mu} e^{-y t-z t^{\mathrm{r}}} J_{v}(x t) d t
\end{aligned}
$$

and (2.2) corresponds to (1.1) and (1.2) and we have

$$
\Omega_{\mu, v}^{\alpha, \beta}(x, y, z \mid 0)=V_{\mu, v}(x, y, z)
$$

and

$$
\begin{aligned}
V_{\mu, v}(x, y, 1 / 4) & =V_{\mu, v}(x, y) \\
& =(2 \sqrt{z})^{\mu+1 / 2} \Omega_{\mu, v}^{\alpha, \beta}(2 x \sqrt{z}, 2 y \sqrt{\mathbf{z}, \mathbf{z} \mid 0})
\end{aligned}
$$

Moreover, $\Omega_{\mu, \nu}^{\alpha, \beta}(x ; y ; 0 \mid 0)$ is the classical Laplace transform of $t^{\mu} J_{v}(x t)$. The case when $z=1 / 4$ and $X=0$ in (2.1) yields

$$
\left.\Omega_{1 / 2,-1 / 2}^{(\alpha, \beta)}\left(x, y, \frac{1}{4} \mid X\right)\right|_{X=0}=
$$

$$
K(x, y) \text { and }\left.\Omega_{1 / 2,1 / 2}^{(\alpha, \beta)}\left(x, y, \frac{1}{4} \mid X\right)\right|_{X=0}=L(x, y)
$$

Using the denition (2.1) with $\alpha=0, \beta=1$ and applying [Prudnikov, et al. (1986), p.581(35)]

$$
{ }_{1} F_{1}(2 ; 1 ; x)=(1+x) e^{x} ;
$$

we get a connection between $V_{\mu, \nu}$ and $\Omega_{\mu, \nu}^{\alpha, \beta}$ in the form

$$
\begin{gathered}
\Omega_{\mu, v}^{0,1}(x ; y ; z \mid X) \\
=V_{\mu, v}(x ; y X ; z X)-X y V_{\mu+1, v}(x ; y X ; z X) \\
-X z V_{\mu+2, v}(x ; y X ; z X)+X^{2} y z V_{\mu+3, v}(x ; y X ; z X)
\end{gathered}
$$

where $V_{\mu, v}$ is given by (1.1).

Similarly setting $\alpha=1, \beta=1$ and applying [Prudnikov, et al. (1986), p.582(53)]

$$
{ }_{1} F_{1}(3 ; 2 ; x)=\frac{(2+x)}{2} e^{x},
$$

we get

$$
\begin{gathered}
\Omega_{\mu, \nu}^{1,1}(x, y, z \mid X)=V_{\mu, \nu}(x, y(1+X), z(1+X))+ \\
\frac{X^{2} y z}{4} V_{\mu+3, \nu}(x, y(1+X), z(1+X)) \\
-\frac{X y}{2} V_{\mu+1, \nu}(x, y(1+X), z(1+X))- \\
\frac{X z}{2} V_{\mu+2, \nu}(x, y(1+X), z(1+X))
\end{gathered}
$$

\section{Explicit Representations for $\Phi_{\mu, v, r}^{\alpha, \beta}$}

In (2.1),we expand ${ }_{1} F_{1}^{\prime s}$ in series and integrate term. We thus find that

$$
\begin{aligned}
& \Phi_{\mu, \nu, r}^{\alpha, \beta}(x, y, z \mid X)= \\
& \sum_{m, n=0}^{\infty} \frac{(\alpha+\beta+1)_{m}(\alpha+\beta+1)_{n} y^{m} z^{n}(-X)^{m+n}}{(\alpha+1)_{m}(\alpha+1)_{n} m ! n !} V_{\mu+m+n r, \nu}(x, y, z)
\end{aligned}
$$

which may be rewritten in the form

$$
\begin{aligned}
& \Phi_{\mu, \nu, r}^{\alpha, \beta}(x, y, z \mid X)=\sum_{m=0}^{\infty} \sum_{n=0}^{\left[\frac{m}{r}\right]} \\
& \frac{(\alpha+\beta+1)_{m-n r}(\alpha+\beta+1)_{n} y^{m-n r} z^{n}(-X)^{m-n r+n}}{(\alpha+1)_{m-n r}(\alpha+1)_{n}(m-n r) ! n !} \\
& V_{\mu+m, \nu, r}(x, y, z)
\end{aligned}
$$

where we have used the series manipulation [Srivastava, et al. (1984), p.101(5)]

$$
\sum_{m, n=0}^{\infty} A(m, n)=\sum_{m=0}^{\infty} \sum_{n=0}^{\left[\frac{m}{r}\right]} A(m, m-n r)
$$

By using a well-known Kummer's theorem [Prudnikov, et al. (1986), p.579(2)]

$$
{ }_{1} F_{1}(a ; b ; x)=e_{1}^{x} F_{1}(b-a ; b ;-x) ;
$$

in (2.1) yields

$$
\begin{aligned}
& \Phi_{\mu, \nu, r}^{\alpha, \beta}(x, y, z \mid X)= \\
& \sum_{m, n=0}^{\infty} \frac{(-\beta)_{m}(-\beta)_{n} y^{m} z^{n}(X)^{m+n}}{(\alpha+1)_{m}(\alpha+1)_{n} m ! n !} \\
& \quad V_{\mu+m+n r, \nu}(x, y(X+1), z(X+1))
\end{aligned}
$$

which further for $X=1$ and $r=2$ reduces to

$$
\begin{aligned}
& \omega_{\mu, \nu}^{\alpha, \beta}(x, y, z)= \\
& \sum_{m, n=0}^{\infty} \frac{(-\beta)_{m}(-\beta)_{n} y^{m} z^{n}}{(\alpha+1)_{m}(\alpha+1)_{n} m ! n !} V_{\mu+m+2 n, \nu}(x, 2 y, 2 z)
\end{aligned}
$$


For $r=2,(3.3)$ reduces to the representation

$$
\begin{aligned}
& \Omega_{\mu, \nu}^{\alpha, \beta}(x, y, z \mid X)= \\
& \sum_{m, n=0}^{\infty} \frac{(-\beta)_{m}(-\beta)_{n} y^{m} z^{n}(X)^{m+n}}{(\alpha+1)_{m}(\alpha+1)_{n} m ! n !} \\
& \quad V_{\mu+m+2 n, \nu}(x, y(X+1), z(X+1))
\end{aligned}
$$

In view of the result (1.7)[Prudnikov, et al. (1986), p.579(18)] with $\beta=n$ (n an integer),(3.4) reduces to

$$
\begin{gathered}
\Omega_{\mu, \nu}^{\alpha, n}(x, y, z \mid X)= \\
\sum_{k, r=0}^{n} \frac{(-(X+1) y)^{k}(-(X+1) z)^{r} n ! n !}{k ! r !(n-k) !(n-r) !(\alpha+1)_{k}(\alpha+1)_{r}} \\
V_{\mu+k+2 r, \nu}(x,(X+1) y,(X+1) z)
\end{gathered}
$$

\section{Series expansions of $\Omega_{\mu, v, r}^{\alpha, \beta}$ involving Jacobi, Laguerre and Hermite polynomials}

We consider the formula [Srivastava, et al. (1984), p.22] expressible in terms of Jacobi polynomials $P_{n}^{(\alpha, \beta)}(x)$ [2] in the form

$$
e_{1}^{t} F_{1}(\alpha+\beta+1, \alpha+1 ;-X t)=\sum_{n=0}^{\infty} \frac{t^{n}}{(\alpha+1)_{n}} P_{n}^{(\alpha, \beta-n)}(1-2 X)
$$

which on replacing $t$ by $y t$ and $t$ by $z t^{r}$ gives

$$
\begin{aligned}
& { }_{1} F_{1}(\alpha+\beta+1, \alpha+1 ;-X y t)= \\
& e^{-y t} \sum_{n=0}^{\infty} \frac{(y t)^{n}}{(\alpha+1)_{n}} P_{n}^{(\alpha, \beta-n)}(1-2 X)
\end{aligned}
$$

and

$$
\begin{aligned}
& { }_{1} F_{1}\left(\alpha+\beta+1, \alpha+1 ;-X z t^{r}\right)= \\
& e^{-z t^{r}} \sum_{n=0}^{\infty} \frac{\left(z t^{r}\right)^{n}}{(\alpha+1)_{n}} P_{n}^{(\alpha, \beta-n)}(1-2 X),
\end{aligned}
$$

respectively. These last two results are now applied to (2.1) to yield a double series representation

$$
\begin{gathered}
\Phi_{\mu, \nu, r}^{\alpha, \beta}=\sum_{m, n=0}^{\infty} \frac{y^{m} z^{r n}}{(\alpha+1)_{m}(\alpha+1)_{n}} P_{m}^{\alpha, \beta-m}(1-2 X) \\
P_{n}^{\alpha, \beta-n}(1-2 X) V_{\mu+m+r n, \nu}(x, y, z)
\end{gathered}
$$

As before, set $\mathrm{r}=2$ and use $\Phi_{\mu, v, 2}^{\alpha, \beta}=\Omega_{\mu, v}^{\alpha, \beta}$ to get

$$
\begin{array}{r}
\Omega_{\mu, \nu}^{\alpha, \beta}=\sum_{m, n=0}^{\infty} \frac{y^{m} z^{2 n}}{(\alpha+1)_{m}(\alpha+1)_{n}} P_{m}^{\alpha, \beta-m}(1-2 X) \\
P_{n}^{\alpha, \beta-n}(1-2 X) V_{\mu+m+2 n, \nu}(x, y, z)
\end{array}
$$

Putting $\mathrm{X}=0$ and using the property $P_{n}^{\alpha, \beta}(1)=\frac{(\alpha+1)_{n}}{n !}$ in (4.2), we obtain the following representation

$$
\begin{aligned}
& V_{\mu, \nu}(x, y, z)= \\
& \sum_{m, n=0}^{\infty} \frac{y^{m} z^{2 n}}{m ! n !} V_{\mu+m+2 n, \nu}(2 x, 2 y, z)
\end{aligned}
$$

Now consider a result [Prudnikov, et al. (1986), p.579 (8)] connecting ${ }_{1} F_{1}$ and Laguerre polynomial

$$
{ }_{1} F_{1}(a, a-n ;-X t)=e^{-X t} \frac{(-1)^{n} n !}{(1-a)_{n}} L_{n}^{(a-n-1)}(X t)
$$

which on replacing $t$ by $y t$ and $t$ by $z t^{r}$ gives

$$
{ }_{1} F_{1}(a, a-n ;-X y t)=e^{-X y t} \frac{(-1)^{n} n !}{(1-a){ }_{n}} L_{n}^{(a-n-1)}(X y t)
$$

and

$$
{ }_{1} F_{1}\left(a, a-n ;-X z t^{r}\right)=e^{-X z t^{r}} \frac{(-1)^{n} n !}{(1-a)_{n}} L_{n}^{(a-n-1)}\left(X z t^{r}\right)
$$

respectively. These last two results are now applied to (2.1) to yield an integral representation

$$
\begin{gathered}
\sqrt{\frac{x}{2}} \int_{0}^{\infty} t^{\mu} e^{-(1+X) y t-(1+X) z t^{r}} L_{n}^{(a-n-1)} \\
(X y t) L_{n}^{(a-n-1)}\left(X z t^{r}\right) J_{\nu}(x t) d t \\
=\frac{(1-a)_{n}(1-a)_{n}}{n ! n !} \Phi_{\mu, \nu, r}^{a-n-1, n}(x,(1-X) y,(1-X) z \mid X)
\end{gathered}
$$

The use of generalized Hermite polynomials defined by (1.8) can be exploited to obtain the series representations of (2.1). We have indeed

$$
\begin{gathered}
\Phi_{\mu, \nu, r}^{\alpha, \beta}=\Phi_{\mu, \nu, r}^{\alpha, \beta}(x, y, z \mid X)=\sqrt{\frac{x}{2}} \sum_{n=0}^{\infty} \frac{1}{n !} H_{n}^{r}(u, v) \\
\int_{0}^{\infty} t^{\mu} e^{-(y+u) t-(z+v) t^{r}{ }_{1} F_{1}(\alpha+\beta+1, \alpha+1 ;-X y t)} \\
{ }_{1} F_{1}\left(\alpha+\beta+1, \alpha+1 ;-X z t^{r}\right) J_{\nu}(x t) d t \\
=\sum_{n=0}^{\infty} \frac{1}{n !} H_{n}^{r}(u, v) \Phi_{\mu+n, \nu, r}^{\alpha, \beta}(x, y+u, z+v \mid X)
\end{gathered}
$$

by applying (1.8) to the integral on the right of (2.1).

Since

$$
\lim _{x \rightarrow 0} x^{-\nu} J_{\nu}(x)=\frac{1}{2^{\nu} \Gamma(\nu+1)}
$$

we may write a limiting case of (2.1) in the form

$$
\begin{gathered}
\lim _{x \rightarrow 0} \frac{\Omega_{\mu, \nu}^{\alpha, \beta}(x, y, z \mid X)}{x^{\nu+1 / 2}}=\frac{2^{\nu+1 / 2}}{\Gamma(\nu+1)} \sum_{n=0}^{\infty} \frac{H_{n}(u, v)}{n !} \\
\int_{0}^{\infty} t^{\mu+\nu+n} e^{-(y+u) t-(z+v) t^{2}}{ }_{1} F_{1}(\alpha+\beta+1, \alpha+1 ;-X y t) \\
{ }_{1} F_{1}\left(\alpha+\beta+1, \alpha+1 ;-X z t^{2}\right) d t
\end{gathered}
$$

which further for $\mathrm{X}=0$ reduces to

$$
\begin{gathered}
\lim _{x \rightarrow 0} \frac{\Omega_{\mu, \nu}^{\alpha, \beta}(x, y, z \mid 0)}{x^{\nu+1 / 2}}=\lim _{x \rightarrow 0} \frac{V_{\mu, \nu}(x, y, z)}{x^{\nu+1 / 2}} \\
=\frac{2^{\nu+1 / 2}}{\Gamma(\nu+1)} \sum_{n=0}^{\infty} \frac{H_{n}(u, v)}{n !} \int_{0}^{\infty} t^{\mu+\nu+n} e^{-(y+u) t-(z+v) t^{2}} d t
\end{gathered}
$$


Now in (4.6), using [Erdelyi, et al. (1954), 146(24)]

$$
\begin{gathered}
\int_{0}^{\infty} t^{\sigma} e^{-y t-z t^{2}} d t=2^{(\sigma+1) / 2} \Gamma(\sigma+1) e^{y^{2} / 8 z} D_{-\sigma-1}\left(\sqrt{\frac{y}{2 z}}\right) \\
(\operatorname{Re}(\sigma+1)>0, \operatorname{Re} y>0)
\end{gathered}
$$

where $D_{-v}(x)$ is parabolic cylinder function [Prudnikov, et al. (1986)], we have

$$
\begin{gathered}
\lim _{x \rightarrow 0} \frac{V_{\mu, \nu}(x, y, z)}{x^{\nu+1 / 2}} \\
=\frac{2^{\nu+1 / 2} e^{(y+u)^{2} / 8(z+v)}}{\Gamma(\nu+1)} \sum_{n=0}^{\infty} \frac{2^{n / 2}(\mu+\nu+1)_{n}}{n !} \\
H_{n}(u, v) D_{-\mu-\nu-n-1}\left(\sqrt{\frac{y+u}{2(z+v)}}\right)
\end{gathered}
$$

A reduction of interest involves the case of replacing $y$ by $y-u, z$ by $z-v$ and $\mu$ by $\mu-v$, and we obtain a known result of Pathan and Shahwan [10] (for $\mathrm{m}=2$ ) in its correct form

$$
\begin{gathered}
\int_{0}^{\infty} t^{\mu} e^{-(y-u) t-(z-v) t^{2}} d t \\
=\Gamma(\mu+1) e^{\frac{y^{2}}{8 z}} \sum_{n=0}^{\infty} \frac{2^{\frac{\mu+n+1}{2}}(\mu+1)_{n}}{n !} \\
H_{n}(u, v) D_{-\mu-n-1}\left(\sqrt{\frac{y}{2 z}}\right)
\end{gathered}
$$

\section{Connections}

We consider the following two integrals

$$
\begin{gathered}
I_{1}=\sqrt{\frac{x}{2}} \int_{0}^{\infty} t^{\mu} e^{-y t-z t^{r}}{ }_{1} F_{1}(\alpha+\beta+1, \alpha+1 ;-X y t) \\
{ }_{1} F_{1}\left(\alpha+\beta+1, \alpha+1 ;-X z t^{r}\right) H_{\nu}(x t) d t
\end{gathered}
$$

where $H_{v}(x)$ are Struve functions [Luke (1969), p.55(8)], $x$, $y, z, X \in \mathbb{R}^{+}, r>1, \operatorname{Re}(\alpha)>-1$ and $\operatorname{Re}(\mu+v)>-1$.

$$
\begin{aligned}
I_{2}= & \sqrt{\frac{x}{2}} \int_{0}^{\infty} t^{\mu} e^{-y t-z t^{r}}{ }_{1} F_{1}(\alpha+\beta+1, \alpha+1 ;-X y t) \\
& { }_{1} F_{1}\left(\alpha+\beta+1, \alpha+1 ;-X z t^{r}\right) s_{\lambda . \nu}(x t) d t
\end{aligned}
$$

where $s_{\lambda . v}(x)$ are Lommel functions [Luke (1969), p.54 (9.4.5) (3)], $x, y, z, X \in \mathbb{R}^{+}, r>1, \operatorname{Re}(\alpha)>-1$ and $\operatorname{Re}(\mu+v)>-1$.

To evaluate these two integrals, we will apply the following two results [Luke (1969), p.55(8)] and [Luke (1969), p.54(9.4.5)(3)]

$$
\begin{gathered}
H_{\nu}(x)=\left(\frac{x}{2 \pi}\right)^{1 / 2} \sum_{n=0}^{\infty} \frac{(x / 2)^{n}}{n !(n+1 / 2)} J_{n+\nu+1 / 2} \\
s_{\lambda . \nu}(x)=\Gamma\left(\frac{\lambda+\nu+1}{2}\right) \frac{x^{(\lambda-\nu+1) / 2}}{2^{(\lambda+\nu-1) / 2}} \\
\sum_{n=0}^{\infty} \frac{(x / 2)^{n}}{n !(2 n+\lambda-\nu+1)} J_{n+(\lambda-\nu+1) / 2}
\end{gathered}
$$

Making appropriate substitution of $H_{v}(x)$ and $s_{\lambda . v}(x)$ from these two results in (5.1) and (5.2), we get

$$
\begin{aligned}
& I_{1}=\left(\frac{x}{2 \pi}\right)^{1 / 2} \sum_{n=0}^{\infty} \frac{x^{n}}{2^{n} n !(n+1 / 2)} \\
& \Phi_{\mu+n+1 / 2, \nu+n+1 / 2, r}^{\alpha, \beta}(x, y, z \mid X)
\end{aligned}
$$

$$
\begin{aligned}
I_{2}= & \Gamma\left(\frac{\lambda+\nu+1}{2}\right) \frac{x^{(\lambda-\nu+1) / 2}}{2^{(\lambda+\nu-1) / 2}} \sum_{n=0}^{\infty} \frac{x^{n}}{2^{n} n !(2 n+\lambda-\nu+1)} \\
& \Phi_{\mu+n+(\lambda-\nu+1) / 2, n+(\lambda-\nu+1) / 2, r}^{\alpha, \beta}(x, y, z \mid X)
\end{aligned}
$$

For $\mathrm{X}=0,(5.1)$ and (5.2) reduce to

$$
\begin{gathered}
\int_{0}^{\infty} t^{\mu} e^{-y t-z t^{r}} H_{\nu}(x t) d t=\left(\frac{x}{2 \pi}\right)^{1 / 2} \\
\sum_{n=0}^{\infty} \frac{x^{n}}{2^{n} n !(n+1 / 2)} V_{\mu+n+1 / 2, \nu+n+1 / 2, r}(x, y, z) \\
\sum_{n=0}^{\infty} \frac{x^{n}}{2^{n} n !(2 n+\lambda-\nu+1)} \\
V_{\mu+n+(\lambda-\nu+1) / 2, n+(\lambda-\nu+1) / 2, r}(x, y, z)
\end{gathered}
$$

Setting $\mathrm{r}=2$ and $z=1 / 4$ in (5.7) and comparing with a known result of [Pathan, et al. (2006), p.78(2.3)], we get

$$
\begin{gathered}
\sqrt{\frac{x}{2}} \int_{0}^{\infty} t^{\mu} e^{-y t-\frac{1}{4} t^{2}}{ }_{1} F_{2}\left(1,3 / 2,1+\nu ;-\frac{x^{2} t^{2}}{4}\right) d t \\
=\left(\frac{x}{2 \pi}\right)^{1 / 2} \sum_{n=0}^{\infty} \frac{x^{n}}{2^{n} n !(n+1 / 2)} V_{\mu+n+1 / 2, \nu+n+1 / 2}(x, y) \\
=\Gamma(\mu+1) x^{\frac{1}{2}} 2^{\frac{\mu}{2}} \sum_{n=0}^{\infty} \frac{(\mu+1)_{n}(-1)^{n} x^{2 n}}{(3 / 2)_{n}(\nu+1)_{n} 2^{n}} \\
e^{\frac{y^{2}}{2}} D_{-\mu-2 n-1}(y \sqrt{2})
\end{gathered}
$$

Setting $\mathrm{r}=2$ in (5.8) and using [Prudnikov, et al. (1986), p.108], we are led to another possibility of dening the Voigt function in the form of Appell function. Thus we have

$$
\begin{gathered}
I_{3}=\int_{0}^{\infty} t^{\mu} e^{-y t-z t^{2}} s_{\lambda, \nu}(x t) d t=\frac{x^{\lambda+1} y^{-a}}{(\lambda+1)^{2}-v^{2}} \sum_{r=0}^{\infty} \frac{(-z)^{r} \Gamma(a+2 r)}{r ! y^{2 r}} \\
{ }_{3} F_{2}\left(1, \frac{a}{2}+r, \frac{a+1}{2}+r ; \frac{3+\lambda-\nu}{2}, \frac{3+\lambda+\nu}{2} ; \frac{-x^{2}}{y^{2}}\right) \\
=\Gamma\left(\frac{\lambda+\nu+1}{2}\right) \frac{x^{(\lambda-\nu) / 2}}{2^{(\lambda+\nu-2) / 2}} \\
\sum_{n=0}^{\infty} \frac{x^{n}}{2^{n} n !(2 n+\lambda-\nu+1)} V_{\mu+n+(\lambda-\nu+1) / 2, n+(\lambda-\nu+1) / 2, r}(x, y, z)
\end{gathered}
$$

where $\alpha=\lambda+\mu+1$. 


\section{Voigt function and numbers}

First we consider a number which we denote by $A_{k}$ with a generating function

$$
\sum_{k=0}^{\infty} A_{k} \frac{t^{k}}{k !}=(1+t)^{-\alpha}\left(1+t^{2}\right)^{-\beta}
$$

The series expansion for $A_{k}$ is

$$
A_{k}=k ! \sum_{m=0}^{\left[\frac{k}{2}\right]} \frac{(-1)^{k+m}(\alpha)_{k-2 m}(\beta)_{m}}{(k-2 m) ! m !}
$$

On comparing (6.1) with (1.14),we find that the number $A_{k}$ and Lagrange-Hermite numbers are related as

$$
A_{k}=k ! h_{k}^{(\alpha, \beta)}(-1,-1)
$$

Moreover from (6.1),we can obtain the following two Laplace transforms

$$
\begin{gathered}
\int_{0}^{\infty} \frac{t^{\mu} e^{-y t} J_{\nu}(x t)}{(1+t)^{\alpha}\left(1+t^{2}\right)^{\beta}} d t \\
=\sum_{k=0}^{\infty} \frac{A_{k}}{k !}\left[\frac{\Gamma(\mu+\nu+k+1)}{y^{\mu+\nu+k+1}}\left(\frac{x}{2}\right)^{\nu}\right. \\
\left.\left(\frac{y^{2}}{x^{2}+y^{2}}\right)^{\mu+\nu+1 / 2}{ }_{2} F_{1}\left(\frac{\nu-\mu-k+1}{2}, \frac{\nu-\mu-k}{2} ; \nu+1 ;-\frac{x^{2}}{y^{2}}\right)\right] \\
\sum_{0=0}^{\infty} \frac{t^{\mu} e^{-y t} \ln t}{(1+t)^{\alpha}\left(1+t^{2}\right)^{\beta}} d t= \\
\sum_{k}^{\infty} \frac{\Gamma(\mu+k+1)}{y^{\mu+k+1}}[\Psi(\mu+k+1)-\ln y]
\end{gathered}
$$

where $\Psi$ is logarithmic derivative of $\Gamma$ function [Andrews, et al. (1999)].

Now we start with a result [Srivastava, et al. (1984), p.84 (15)] for Laguerre polynomials

$$
e^{-y t}=(1+t)^{-\alpha} \sum_{n=0}^{\infty} L_{n}^{(\alpha-n)}(y) t^{n}
$$

which on replacing $t$ by $t^{2}, \alpha$ by $\beta$ and $y$ by $z$ gives

$$
e^{-z t^{2}}=\left(1+t^{2}\right)^{-\beta} \sum_{m=0}^{\infty} L_{m}^{(\beta-m)}(z) t^{2}
$$

On multiplying these two results yields

$$
\begin{aligned}
& e^{-y t-z t^{2}}=(1+t)^{-\alpha}\left(1+t^{2}\right)^{-\beta} \\
& \sum_{n, m=0}^{\infty} L_{n}^{(\alpha-n)}(y) L_{m}^{(\beta-m)}(z) t^{n+2 m}
\end{aligned}
$$

which is equivalent to

$$
\frac{e^{-y t-z t^{2}}}{(1+t)^{-\alpha}\left(1+t^{2}\right)^{-\beta}}=\sum_{n, m=0}^{\infty} L_{n}^{(\alpha-n)}(y) L_{m}^{(\beta-m)}(z) t^{n+2 m} \text { (6.5) }
$$

Using (6.1) and (1.10) in (6.4) gives

$$
\begin{aligned}
& \sum_{n=0}^{\infty} H_{n}(-y,-z) \frac{t^{n}}{n !}= \\
& \sum_{k=0}^{\infty} A_{k} \frac{t^{k}}{k !} \sum_{n, m=0}^{\infty} L_{n}^{(\alpha-n)}(y) L_{m}^{(\beta-m)}(z) t^{n+2 m}
\end{aligned}
$$

Comparing the coecients of $t^{n}$ on both the sides of (6.6), we get the the following representation of Hermite polynomials in the form

$$
\begin{aligned}
& H_{n}(-y,-z)= \\
& \quad n ! \sum_{k=0}^{n} \frac{A_{k}}{k !} \sum_{m=0}^{\left[\frac{n-m}{2}\right]} L_{n-k-2 m}^{(\alpha-n-k-2 m)}(y) L_{m}^{(\beta-m)}(z)
\end{aligned}
$$

In view of the result (1.12) expressed for Hermite numbers $H_{n}$, for $y=z=0,(6.7)$ gives

$$
\begin{gathered}
H_{n}=\left\{\begin{array}{l}
0, \quad \text { if } \mathrm{n} \text { is odd } \\
\frac{(-1)^{n / 2} n !}{\left(\frac{n}{2}\right) !}, \text { if } \mathrm{n} \text { is even }
\end{array}\right. \\
=\left\{\begin{array}{l}
0, \quad \text { if } \mathrm{n} \text { is odd } \\
\sum_{k=0}^{n} \frac{A_{k}}{k !} \sum_{m=0}^{\left[\frac{n}{2}\right]} \frac{(\alpha-n-k-2 m+1)_{n}(\beta-m+1)_{m}}{m !}, \text { if } \mathrm{n} \text { is even }
\end{array}\right.
\end{gathered}
$$

Now we turn to the derivation of the representation of voigt function from (6.7). Multiply both he sides of (6.4) by $t^{\mu} e^{-y 1 t-z 1 t^{2}} J_{v}(x t)$ and integrate with respect to $t$ from 0 to $\infty$ to get

$$
\begin{aligned}
& V_{\mu, \nu}\left(x, y+y_{1}, z+z_{1}\right)= \\
& \sqrt{\frac{x}{2}} \sum_{n, m=0}^{\infty} L_{n}^{(\alpha-n)}(y) L_{m}^{(\beta-m)}(z) \\
& \int_{0}^{\infty} \frac{t^{\mu+n+2 m} e^{-y_{1} t-z_{1} t^{2}} J_{\nu}(x t)}{(1+t)^{\alpha}\left(1+t^{2}\right)^{\beta}} d t
\end{aligned}
$$

which on using (6.1) gives

$$
\begin{aligned}
& V_{\mu, \nu}\left(x, y+y_{1}, z+z_{1}\right)= \\
& \sum_{k, n, m=0}^{\infty} \frac{A_{k}}{k !} L_{n}^{(\alpha-n)}(y) L_{m}^{(\beta-m)}(z) V_{\mu+n+k+2 m, \nu}\left(x, y_{1}, z_{1}\right)
\end{aligned}
$$

For $y=z=0,(6.9)$ gives an interesting relation between Voigt functions in the form

$$
\begin{gathered}
V_{\mu, \nu}\left(x, y_{1}, z_{1}\right)= \\
\sum_{k, n, m=0}^{\infty} \frac{A_{k}}{k !} \frac{(\alpha-n+1)_{n}(\beta-m+1)_{m}}{n ! m !} \\
V_{\mu+n+k+2 m, \nu}\left(x, y_{1}, z_{1}\right)
\end{gathered}
$$

Yet, another immediate consequence of (6.9) is obtained by taking $y 1=y=z 1=0$ and applying (6.2). Thus we have 


$$
\begin{gathered}
V_{\mu, \nu}(x, 2 y, z)=\left(\frac{x}{2}\right)^{\nu+1 / 2} \\
\sum_{n, m, k=0}^{\infty} L_{n}^{(\alpha-n)}(y) L_{m}^{(\beta-m)}(z) \frac{A_{k}}{k !}\left[\frac{\Gamma(\mu+n+2 m+\nu+k+1)}{y^{\mu+n+2 m+\nu+k+1}}\right. \\
\left(\frac{y^{2}}{x^{2}+y^{2}}\right)^{\mu+n+2 m+\nu+1 / 2} \\
\left.{ }_{2} F_{1}\left(\frac{\nu-\mu-n-2 m-k+1}{2}, \frac{\nu-\mu-n-2 m-k}{2} ; \nu+1 ;-\frac{x^{2}}{y^{2}}\right)\right]
\end{gathered}
$$

By setting $\mathrm{z}=0$ in (6.4) and multiplying both he sides by $t^{\mu} e^{-y t} \ln t$, integrating with respect to $\mathrm{t}$ from 0 to $\infty$ and using (6.3) and [Erdelyi, et al. (1954), p.148(4)]

$$
\int_{0}^{\infty} t^{\mu} e^{-y t} \ln t d t=\frac{\Gamma(\mu+1)}{y^{\mu+1}}[\Psi(\mu+1)-\ln y]
$$

we get

$$
\begin{gathered}
\sum_{k, n, m=0}^{\infty} \frac{A_{k}(\beta-m+1)_{m}}{k ! m !} \frac{(\mu+1)_{n+2 m+k}}{y^{n+2 m+k}} L_{n}^{(\alpha-n)}(y) \\
{[\Psi(\mu+n+2 m+k+1)-\ln y]} \\
=[\Psi(\mu+1)-\ln 2 y]
\end{gathered}
$$

where $\Psi$ is logarithmic derivative of $\Gamma$ function [Srivastava, et al. (1984)].

If, in (6.5),we set $\alpha=\beta=1$, multiply both he sides by $t^{\mu} e^{-y t-z t^{2}} J_{v}(x t)$ and integrate with respect to $\mathrm{t}$ from 0 to $\infty$, we get a generalization of (6.10) in the form

$$
\begin{gathered}
\sum_{n, m=0}^{\infty} L_{n}^{(1-n)}(y) L_{m}^{(1-m)}(z) V_{\mu+n+2 m, \nu}(x, y, z) \\
\quad=V_{\mu, \nu}(x, 2 y, 2 z)+V_{\mu+1, \nu}(x, 2 y, 2 z) \\
+V_{\mu+2, \nu}(x, 2 y, 2 z)+V_{\mu+3, \nu}(x, 2 y, 2 z)
\end{gathered}
$$

\section{Some useful consequences of Lagarange-Hermite polynomials.}

Now we start with a result [Srivastava, et al. (1984), p.84 (15)] for Laguerre polynomials written in a slightly different form

$$
e^{-x_{1} y t}=\left(1-x_{1} t\right)^{-\alpha} \sum_{n=0}^{\infty} L_{n}^{(\alpha-n)}(y)\left(-x_{1} t\right)^{n}
$$

which on replacing $t$ by $t^{2}, \alpha$ by $\beta, x 1$ by $x 1$ and $y$ by $z$ gives

$$
e^{-x_{2} z t^{2}}=\left(1-x_{2} t^{2}\right)^{-\beta} \sum_{m=0}^{\infty} L_{m}^{(\beta-m)}(z)\left(-x_{2} t^{2}\right)^{m}
$$

On multiplying these two results and adjusting the variables yields

$$
\begin{aligned}
e^{y t+z t^{2}}= & \left(1-x_{1} t\right)^{-\alpha}\left(1-x_{2} t^{2}\right)^{-\beta} \\
& \sum_{n, m=0}^{\infty}(-1)^{n+m} x_{1}^{n} x_{2}^{m} L_{n}^{(\alpha-n)}\left(\frac{y}{x_{1}}\right) L_{m}^{(\beta-m)}\left(\frac{z}{x_{2}}\right) t^{n+2 m}
\end{aligned}
$$

which is equivalent to

$$
\begin{aligned}
1= & \frac{e^{-y t-z t^{2}}}{\left(1-x_{1} t\right)^{\alpha}\left(1-x_{2} t^{2}\right)^{\beta}} \\
& \sum_{n, m=0}^{\infty}(-1)^{n+m} x_{1}^{n} x_{2}^{m} L_{n}^{(\alpha-n)}\left(\frac{y}{x_{1}}\right) L_{m}^{(\beta-m)}\left(\frac{z}{x_{2}}\right) t^{n+2 m}
\end{aligned}
$$

Using the definition of Lagrange-Hermite polynomials $h_{k}^{\left(\alpha_{1}, \alpha_{2}\right)}\left(x_{1}, x_{2}\right)$ given by (1.14) in (7.2), we get

$$
\begin{array}{r}
1=e^{-y t-z t^{2}} \sum_{k, n, m=0}^{\infty}(-1)^{n+m} x_{1}^{n} x_{2}^{m} h_{k}^{(\alpha, \beta)} \\
\left(x_{1}, x_{2}\right) L_{n}^{(\alpha-n)}\left(\frac{y}{x_{1}}\right) L_{m}^{(\beta-m)}\left(\frac{z}{x_{2}}\right) t^{k+n+2 m}
\end{array}
$$

which on replacing $n$ by $n-2 m$ gives

$$
\begin{aligned}
e^{y t+z t^{2}}= & \sum_{k, n=0}^{\infty} \sum_{m=0}^{\left[\frac{n}{2}\right]}(-1)^{n-m} x_{1}^{n-2 m} x_{2}^{m} h_{n-2 m}^{(\alpha, \beta)} \\
& \left(x_{1}, x_{2}\right) L_{n-2 m}^{(\alpha-n-2 m)}\left(\frac{y}{x_{1}}\right) L_{m}^{(\beta-m)}\left(\frac{z}{x_{2}}\right) t^{k+n}
\end{aligned}
$$

Again applying the denition of Hermite polynomials given by (1.10) in (7.4), replacing $n$ by $n-k$ and comparing the coecients of $t^{n}$, we get the following representation of $H_{n}(y, z)$

$$
\begin{aligned}
H_{n}(y, z)= & n ! \sum_{k=0}^{n} \sum_{m=0}^{\left[\frac{n-k}{2}\right]}(-1)^{n-k-m} x_{1}^{n-k-2 m} x_{2}^{m} h_{n-k-2 m}^{(\alpha, \beta)} \\
& \left(x_{1}, x_{2}\right) L_{n-k-2 m}^{(\alpha-n+k-2 m)}\left(\frac{y}{x_{1}}\right) L_{m}^{(\beta-m)}\left(\frac{z}{x_{2}}\right)
\end{aligned}
$$

which reduces to (6.7) when we take $x 1=x 2=1$ and use $A_{k}$ $=k ! h_{k}^{(\alpha, \beta)}(-1,-1)$.

It is also fairly straightforward to get a representation of generalized Voigt function $V_{\mu, v}$ by appealing (7.3). We multiply both he sides by $t^{\mu} e^{-y 1 t-z 1 t^{2}} J_{v}(x t)$ and integrate with respect to $t$ from 0 to $\infty$. Thus we get

$$
\begin{aligned}
V_{\mu, \nu}\left(x, y_{1}, z_{1}\right)= & \\
& \sum_{k, n, m=0}^{\infty}(-1)^{n+m} x_{1}^{n} x_{2}^{m} h_{k}^{(\alpha, \beta)}\left(x_{1}, x_{2}\right) \\
& L_{n}^{(\alpha-n)}\left(\frac{y}{x_{1}}\right) L_{m}^{(\beta-m)} \\
& \left(\frac{z}{x_{2}}\right) V_{\mu+k+n+2 m, \nu}\left(x, y+y_{1}, z+z_{1}\right)
\end{aligned}
$$

On the other hand, multiplying both the sides of (7.4) by

$$
\begin{aligned}
t^{\mu} e_{1}^{-2 y t-2 z t^{2}} F_{1}(\alpha+\beta+1, \alpha+1 ;-X y t) \\
{ }_{1} F_{1}\left(\alpha+\beta+1, \alpha+1 ;-X z t^{r}\right) J_{\nu}(x t)
\end{aligned}
$$


and integrating with respect to $\mathrm{t}$ from 0 to $\infty$ and then using (2.2), we get a generalization of (6.10) in the form

$$
\begin{aligned}
& \Omega_{\mu, \nu}^{\alpha, \beta}(x, y, z \mid X)= \\
& \sum_{k, n=0}^{\infty} \sum_{m=0}^{\left[\frac{n}{2}\right]}(-1)^{n-m} x_{1}^{n-2 m} x_{2}^{m} h_{n-2 m}^{(\alpha, \beta)}\left(x_{1}, x_{2}\right) \\
& L_{n-2 m}^{(\alpha-n-2 m)}\left(\frac{y}{x_{1}}\right) L_{m}^{(\beta-m)}\left(\frac{z}{x_{2}}\right) \Omega_{\mu+k+n, \nu}^{\alpha, \beta}\left(x, 2 y, 2 z \mid \frac{1}{2} X\right)
\end{aligned}
$$

\section{References}

Altin, A, Erkus, E. (2006). On a multivariable extension of the Lagrange-Hermite polynomials, Integral Transforms Spec. Funct., 17: 239-244.

Andrews, GE, Askey, R, Roy, R. (1999). Special Functions. Cambridge University Press, Cambridge.

Chan, WChC, Chyan, ChJ, Srivastava, HM. (2001). The Lagrange Polynomials in Several Variables, Integral Transforms Spec. Funct. 12 (2): 139-148.

Dattoil, G, Ricci, PE, Cesarano, C. (2003). The Lagrange polynomials the associated generalizations, and the umbral calculus, Integral Transforms Spec. Funct. 14: 181-186.

Erdelyi, A. et al. (1954). Tables of Integral Transforms, Vol. I. Mc Graw Hill, New York, Toronto, London.
Gould, HW, Hopper, AT. (1962). Operational formulas connected with two generalizations of Hermite polynomials, Duke Math. J. 29: 51-63.

Klusch, D. (1991). Astrophysical Spectroscopy and neutron reactions, Integral transforms and Voigt functions, Astrophys. Space Sci. 175: 229-240.

Luke, YL. (1969). The Special Functions and their approximations, Academic Press, New York, London.

Pathan, MA, Kamarujjama, M, Khursheed Alam M. (2003). Multiindices and multivariable presentations of Voigt Functions, J. Comput. Appl. Math. 160: 251-257.

Pathan, MA, Shahwan, MJS. (2006). New representations of the Voigt Functions, Demonstatio Math. 39: 75-80.

Prudnikov, AP, et al. (1986). Integral and Series, Vol. 2, Special Functions, Gorden and Breech Sciences Publisher, New York.

Srivastava, HM, Joshi, CM. (1967). Certain double Whittaker transforms of generalized hypergeometric functions, Yokohama Math. J. 15: 19-31.

Srivastava, HM, Manocha, HL. (1984). A Treatise on Generating Functions, Ellis Horwood Limited, Chichester.

Srivastava, HM, Miller, EA. (1987). A Unied presentation of the Voigt functions, Astrophys Space Sci. 135: 111-115.

Srivastava, HM, Pathan, MA, Kamarujjama, M. (1998). Some unied presentations of the generalized Voigt functions, Comm. Appl. Anal. 2: 49-64.

Yang S. (1994). A unication of the Voigt functions,Int.J.Math. Educ.Sci.Technol. 25: 845-851. 